\title{
Authors' Reply: The Russian gonococcal antimicrobial susceptibility programme (RU-GASP)--national resistance prevalence in 2007 and 2008, and trends during 2005-2008
}

\author{
M Unemo (magnus.unemo@orebroll.se)1', M Domeika² \\ 1. National Reference Laboratory for Pathogenic Neisseria, Department of Laboratory Medicine, Clinical Microbiology, Örebro \\ University Hospital, Örebro, Sweden \\ 2. Department of Medical Sciences, Uppsala University, Uppsala, Sweden
}

Citation style for this article:

Citation style for this article: Unemo M, Domeika M. AUTHORS' REPLY: The Russian gonococcal antimicrobial susceptibility programme (RU-GASP)--national

resistance prevalence in 2007 and 2008, and trends during 2005-2008. Euro Surveill. 2010;15(19):pii=19569. Available online: http://www.eurosurveillance.org/

ViewArticle.aspx?Articleld $=19569$

This article has been published on 13 May 2010

To the editor: We are grateful to Gil-Setas et al. for their comments on our paper [1]. In their letter "Spectinomycin in the treatment of gonorrhoea", they further clarify and emphasise the importance of, if possible, avoiding spectinomycin in the treatment of pharyngeal gonorrhoea, which is an important comment. Spectinomycin is an effective injectable antimicrobial [2] and is useful for treatment of patients with uncomplicated anogenital gonorrhoea who, for example, cannot tolerate extended-spectrum cephalosporins or if extended-spectrum cephalosporins are not available. Fluoroquinolones have also been used in the past, but currently these are of limited importance for empirical treatment in most settings worldwide due to a high level of resistance [3]. However, it has been known for decades that spectinomycin is suboptimal (with sometimes as low as approximately 50\% effectiveness) for treatment of pharyngeal infections, due to the pharmacokinetic properties of spectinomycin [2,4-7]. This lower efficacy in eradicating Neisseria gonorrhoeae from the pharynx, compared with eradication of the bacteria in anogenital infection, is also a problem for several other antimicrobials $[2,3,7]$, and it would be valuable to assess this issue in additional studies. In fact, among the antimicrobials available for treatment of uncomplicated gonococcal, including pharyngeal, infections, extended-spectrum cephalosporins and fluoroquinolones seem to have the best balance of proven efficacy and safety for wild-type, susceptible gonococci. However, as mentioned above fluoroquinolones are not recommended for use in most settings due to the high level of resistance $[2,7]$.

It is important to note that pharyngeal gonococcal infection, solely or combined with anogenital infection, is not extremely rare and prevalence may have increased over time, perhaps due to changes in sexual behaviours [8]. Accordingly, some patients treated for anogenital gonorrhoea may also have a coincident pharyngeal infection, which must also be treated. If spectinomycin is the only option for empirical treatment - due to unavailability of ceftriaxone and a high level of resistance to fluoroquinolones, or severe allergy to beta-lactam antimicrobials [rare cases] and in vitro resistance to all other treatment options - it is recommended that all patients with suspected (after a risk assessment has been performed) or confirmed pharyngeal infection have a pharyngeal culture taken approximately 3-5 days after treatment, i.e., as a testof-cure to verify effective eradication of the gonococci.

Obviously, we totally agree with the warning of GilSetas et al. about the potential risks of using spectinomycin for treatment of pharyngeal gonorrhoea. Nevertheless, the recommendations for first-line empirical treatment described in our paper [1] are mainly for uncomplicated gonococcal infections of the urethra, cervix, and rectum (anogenital gonorrhoea) in adults and adolescents. In this type of paper, due to the limit in word count, it is not possible to separate all indications for treatment and discuss the pros and cons of each treatment (including the pharmacokinetic properties of the drugs) as described in different treatment guidelines. In addition to pharyngeal gonorrhoea, the indications also include other extra-genital gonococcal infections, different types of complications and sequelae, disseminated gonococcal infection (DGI), etc. All treatment indications related to gonorrhoea and their treatment options are thoroughly described in the Russian treatment guidelines. As in other guidelines, such as those published by the United States Centers for Disease Control and Prevention (CDC) $[5,6]$ and the International Union against Sexually Transmitted Infections (IUSTI) [9], the Russian treatment guidelines do not recommend spectinomycin for treatment of pharyngeal gonorrhoea if other effective antimicrobials are available. 
Regarding the use of azithromycin (single oral dose of $2 \mathrm{~g}$ ) for treatment of anogenital as well as pharyngeal gonorrhoea, this is effective for wild-type, susceptible gonococci. However, using the azithromycin formula presently available in most countries, substantial gastrointestinal side effects may occur and this treatment option can rapidly select for resistance. Increasing resistance to azithromycin, including highlevel resistance, has been identified in many countries [3]. Accordingly, this treatment option should not be widely recommended, i.e. if it is not used in a strictly controlled manner and the in vitro susceptibility to azithromycin has been determined before treatment.

One additional important comment is that although resistance to spectinomycin has remained rare in most countries, rapid selection of resistance was reported as early as the 1980 [ [10]. Accordingly, if spectinomycin is used for treatment of gonorrhoea, it has to be used in a strictly controlled manner and effective monitoring of its quality, use, as well as emergence and spread of resistance is essential.

In conclusion, the gonococci have developed resistance to almost all antimicrobials used in the treatment of gonorrhoea. At present, the first-line treatment in most countries is extended-spectrum cephalosporins such as cefixime (oral) and ceftriaxone (injectable). However, the susceptibility to all extended-spectrum cephalosporins is decreasing and treatment failures using oral ones have been identified in several settings [3]. If clinical resistance emerges also to ceftriaxone, gonorrhoea may become untreatable in certain circumstances. Accordingly, treatment options have to be available for these emergent situations, and having access to antimicrobials such as spectinomycin, which at present is not available in many countries, would be extremely valuable.
7. Moran JS. Treating uncomplicated Neisseria gonorrhoeae infections: is the anatomic site of infection important? Sex Transm Dis. 1995;22(1):39-47.

8. Janier M, Lassau F, Casin I, Morel P. Pharyngeal gonorrhoea: the forgotten reservoir. Sex Transm Infect. 2003;79(4):345.

9. Bignell C; IUSTI/WHO. 2009 European (IUSTI/WHO) guideline on the diagnosis and treatment of gonorrhoea in adults. Int J STD AIDS. 2009;20(7):453-7.

10. Boslego JW, Tramont EC, Takafuji ET, Diniega BM, Mitchell BS, Small JW, et al. Effect of spectinomycin use on the prevalence of spectinomycin-resistant and penicillinase-producing Neisseria gonorrhoeae. N Engl J Med. 1987;317(5):272-8.

\section{References}

1. Kubanova A, Frigo N, Kubanov A, Sidorenko S, Lesnaya I, Polevshikova S, et al. The Russian gonococcal antimicrobial susceptibility programme (RU-GASP)--national resistance prevalence in 2007 and 2008, and trends during 2005-2008. Euro Surveill. 2010;15(14). pii=19533. Available from: http:// www.eurosurveillance.org/ViewArticle.aspx?Articleld $=19533$

2. Moran JS, Levine WC. Drugs of choice for the treatment of uncomplicated gonococcal infections. Clin Infect Dis. 1995;20 Suppl 1:S47-65.

3. Tapsall JW, Ndowa F, Lewis D, Unemo M. Meeting the public health challenge of multi- and extensively-drug resistant Neisseria gonorrhoeae. Expert Rev Anti Infect Ther. 2009;7(7):821-34.

4. Lindberg M, Ringertz O, Sandström E. Treatment of pharyngeal gonorrhoea due to beta-lactamase-producing gonococci. $\mathrm{Br}$ J Vener Dis. 1982;58(2):101-4.

5. Centers for Disease Control and Prevention, Workowski KA, Berman SM. Sexually transmitted diseases treatment guidelines, 2006. MMWR Recomm Rep. 2006:55(RR-11);194. Available from: http://www.cdc.gov/mmwr/preview/ mmwrhtml/rr5511a1.htm

6. Centers for Disease Control and Prevention (CDC). Update to CDC's sexually transmitted diseases treatment guidelines, 2006: fluoroquinolones no longer recommended for treatment of gonococcal infections. MMWR Morb Mortal Wkly Rep. 2007;56(14):332-6. Available from: http://www.cdc.gov/mmwr/ preview/mmwrhtml/mm5614a3.htm. 